\title{
Identificación de poblaciones de vid silvestre y parras guiadas sobre las fachadas de los caseríos de la Cuadrilla de Ayala (Álava, España).
}

\section{Identifying populations of wild vines and grapevi- nes trained on the facades of old farmhouses.}

Juanjo Hidalgo ${ }^{1 *}$, José Miguel Martínez-Zapater ${ }^{2}$, Rafael Ocete ${ }^{3}$, Miguel Lara ${ }^{4}$, Álvaro Rodríguez ${ }^{5}$, José Manuel Valle ${ }^{5}$, Ramón Morales ${ }^{6}$, María José Iriarte-Chiapusso ${ }^{7}$, Carlos Alvar-Ocete ${ }^{3}$ y Javier Ibáñez ${ }^{2}$

\section{(*}

\section{Resumen}

El presente trabajo se ha centrado sobre la Cuadrilla de Ayala (Álava), donde se han localizado ocho poblaciones relictas de vid silvestre en diversos hábitats. Se ha comprobado su carácter dioico durante el periodo de floración. Se han identificado las principales especies parásitas existentes sobre estas parras y las principales componentes de su vegetación acompañante. Por otro lado, se han estudiado quince vides cultivadas, con una edad supe-

\footnotetext{
1 Laudioalde Lanbide Eskola \& Aunia Kultura Elkartea. Llodio, Álava.

*Correspondencia: juanjoaunia@gmail.com

2 Instituto de Ciencias de la Vid y del Vino (CSIC),

Gobierno de La Rioja, Universidad de La Rioja. Logroño.

3 Laboratorio de Entomología Aplicada, Facultad de Biología. Universidad de Sevilla.

4 IFAPA, Rancho de la Merced. Jerez de la Frontera, Cádiz.

5 Escuela Universitaria de Ingeniería, Universidad del País Vasco. Vitoria-Gasteiz.

6 Real Jardín Botánico (CSIC). Madrid.

7 Área de Prehistoria, IKERBASQUE Universidad del País Vasco. Vitoria-Gasteiz.
} 
rior a cincuenta años, situadas en la fachada principal de los caseríos. El análisis del ADN de ambos tipos de parras se ha realizado con 48 marcadores SNPs. Los resultados obtenidos han sido comparados con la base de datos disponible en el Instituto de Ciencias de la Vid y del Vino (Logroño), a fin de proceder a la caracterización genética de las muestras.

Palabras clave: Chacolí, estado sanitario, marcadores SNP, vegetación acompañante, Vitis vinifera subsp. sylvestris (C.C. Gmelin) Beger \& Hegi.

\begin{abstract}
This paper focuses on the Cuadrilla de Ayala (Álava province, Spain), where eight relic wild grapevine populations were located in different habitats. The dioecious nature of these lianas was determined at flowering time. The main parasitic species on these vines and also the most frequent taxa of their accompanying vegetation were identified. On the other hand, fifteen vines, aged in excess of fifty, and located in the main entrances of traditional farmhouses (caseríos), were also studied. The analysis of the DNA from both kinds of sampled vines was based on 48 SNPs markers. Results were compared with the available data base at the Instituto de Ciencias de la Vid y del Vino (Logroño, Spain) for the sample genetic characterization.
\end{abstract}

Key words: Chacolí wine, SNP markers, sanitary status, accompanying vegetation, Vitis vinifera L. subsp. sylvestris (C.C. Gmelin) Beger \& Hegi.

\title{
Laburpena
}

Honako lan hau Aiaraldean (Araba) kokatu da. Habitat desberdinetan bizi diren zortzi basamahats-gune aurkitu dira, desagertzeko puntuan. Loraldian dioikoak direla egiaztatu da. Mahats-parra horietan dauden espezie parasito gehienak identifikatu dira eta bai beraiekin batera bizi diren gainerako landare laguntzaileak ere.

Bestalde, hamabost mahats-landare landu ikertu dira, berrogeita hamar urtetik gorakoak, baserrietako aurrealdeko horman hazi direnak. Bi mahatsondoen ADN analisia SNP 48 markatzailerekin egin da. Lortutako emaitzak Logroñoko Instituto de Ciencias de la Vid y del Vino-ko datu-basearekin alderatu dira, laginen ezaugarri genetikoak zehazteko.

Gako hitzak: txakolina, osasun egoera, SPN markatzaileak, landaredi laguntzailea, Vitis vinifera subsp. sylvestris (C.C. Gmelin) Beger \& Hegi. 


\section{Introducción}

El taxón Vitis vinifera L. subsp. sylvestris (C.C. Gmelin) Beger \& Hegi constituye la denominada vid silvestre euroasiática. Sus poblaciones están integradas por lianas que toman por tutor a la vegetación circundante. Éstas se extienden desde la Península Ibérica hasta Afganistán, y también en algunas zonas de Magreb. Entre los paralelos 30 (Río Ourika, Marruecos) y 50 (Río Rin, Alemania) (Morales \& Ocete, 2015). Dicha subespecie constituye el parental dioico de las variedades de cultivo, englobadas en la subespecie sativa, integrada mayoritariamente por cultivares hermafroditas seleccionados por el ser humano (Arnold, 2002).

Parece ser que la Península Ibérica fue uno de los refugios europeos para la vid silvestre durante el Pleistoceno. Tras la finalización del último periodo glaciar, las nuevas condiciones medioambientales y la expansión de los bosques meso-termófilos facilitaron la recuperación de la vid silvestre y otras especies botánicas en la Península Ibérica (Iriarte-Chiapusso et al., 2005). Dentro del País Vasco, aparecen granos de polen de esta vitácea en la zona de Urdaibai (Bizkaia) correspondientes a los comienzos del Holoceno, concretamente del décimo milenio antes del presente (Iriarte-Chiapusso et al., 2006).

Las vides silvestres han tenido diversos usos para los humanos: madera, maromas, nasas de pesca, producción de vino y vinagre, o medicamentos, entre otros. (Ocete et al. 2011a). Dichas parras constituyen, actualmente, un importante recurso fitogenético de cara a la mejora de las variedades de cultivo frente a la problemática del cambio climático. Además son las antecesoras de más del 70\% de las variedades de cultivo españolas (con genoma cloroplástico o clorotipo A). Sin embargo, las variedades silvestres y cultivadas de Transcaucasia contienen el C y D (Arroyo-García et al., 2006). Hay que tener en cuenta que las semillas de vid cultivada más antiguas, encontradas hasta ahora, de 8.000 años a.C., proceden del yacimiento arqueológico de Shulaveri Goraen Georgia (Chilashvili, 2004).

En el País Vasco, algunas poblaciones fueron descritas en Bizkaia por Guinea (1949). Actualmente, las poblaciones relictas de vid silvestre se encuentran en bosques de ribera, en posición coluvial en las faldas de montes y colinas del interior de su territorio, así como sobre acantilados litorales. Los nombres que reciben las parras en el mundo rural vasco son basamahatsondo (vid silvestre), zozo-mahatsa (uva de tordo) y uvillas (los ejemplares femeninos) (Ocete et al., 2004).

El cultivo de diversos frutales y la vid llegaron a la Península Ibérica de la mano de los fenicios y colonizadores griegos, romanos, cartagineses y árabes. El consumo de muchos de estos frutos por parte del ejército romano y la generalización del comercio tras la pacificación debió de influir en la propagación de los mismos en el territorio vasco, tal y como se ha constatado en el yacimiento guipuzcoano del puerto de la antigua ciudad de Oiasso (Irún). En el mismo, se han encontrado semillas cultivadas 
de vid, datadas en el siglo I d.C. Resulta realmente difícil saber si dichas pepitas corresponden a plantas cultivadas en el ámbito vasco o a uvas importadas mediante el comercio, lo que parece más probable (Zapata, 2002).

La documentación existente sobre viñas y vino en el País Vasco durante la Alta y Baja Edad Media es bastante amplia. Cartularios y becerros como los de San Millán de la Cogolla, Valpuesta, Eslonza, Oña, y las fuentes documentales medievales aportan datos de gran interés histórico. Dicha documentación aún se hará más abundante con la llegada de la Edad Moderna gracias a las recurridas ordenanzas municipales de las villas, que trataban de regular toda la actividad referida a la vitivinicultura, desde el cultivo hasta la venta del propio vino (Hidalgo \& Llano, 2007).

La Cuadrilla de Ayala o Airaraldea, constituye una de las siete demarcaciones administrativas en las que se divide la provincia de Álava. Esta comarca limita con las provincias de Bizkaia y Burgos y constituye el centro de la actual denominación de origen Chacolí de Álava / Arabako Txakolina (Figura 1).

Esta zona ha sido productora de vino desde la Alta Edad Media, vinculada por entonces al Monasterio de Valpuesta. En el Cartulario de dicho monasterio existe un docu-

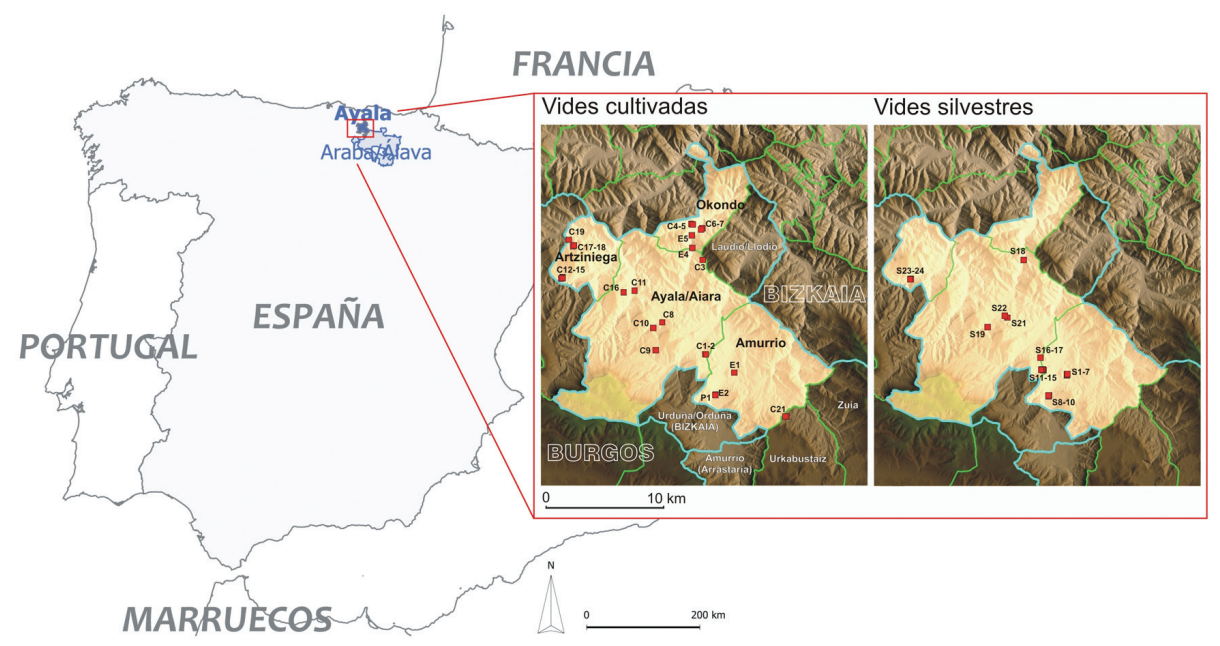

Fig. 1.- Mapa de situación de la Tierra de Ayala en la provincia de Álava y en España. Localización de las muestras de parras cultivadas recogidas en fachadas de los caseríos de la Cuadrilla de Ayala, así como de las silvestres, para el presente trabajo.

Fig. 1.- Map of the situation of the Land of Ayala in the province of Álava and in Spain. Location of the samples of cultivated vines collected on the facades of the farmhouses of Cuadrilla de Ayala, as well as of wild vines, for the present study. 
mento del año 864, que hace referencia a donaciones de viñas realizadas por Elduara y sus hijos a la Iglesia de Santa María, en Retes de Tudela, Artziniega. Entre las donaciones aparecen pommares (manzanares), terras sationavilis (tierras de sembrar) y vineas (viñas) (Pérez Soler, 1970). Asimismo, en el Cartulario de San Millán de la Cogolla, existe otro documento del año 964, donde se recoge la donación de distintos bienes procedentes del monasterio de San Víctor y Santiago, asentado en Gardea (Llodio), al monasterio de San Esteban y Santa María de Salcedo. Entre los lotes asignados, se hace referencia, igualmente, a las viñas (Ubieto, 1976).

Durante el Medioevo, la importancia del vino como fuente calórica y litúrgica hizo que el viñedo estuviese bien protegido por la legislación. Así, en el Fuero Viejo de Bizkaia (1454), se recoge la pena de muerte a aquellas personas que arrancasen cinco cepas en viñedo ajeno, y otras sanciones por los daños causados por el ganado. Asimismo, las ordenanzas municipales, hasta finales del siglo XVIII, solían contener prohibiciones sobre la entrada de vino foráneo, como el de La Rioja, hasta no haberse consumido el local. A este vino de la tierra, se le fue dando el nombre de "Chacolín", a partir del siglo XVI (Hidalgo \& Llano, 2007).

Los chacolís, eran lugares donde se producía el vino, se vendía y consumía, eran oasis de esparcimiento donde tomar una jarra o porrón de vino de la tierra, donde echar una tirada de bolos, y donde cantar y merendar a la sombra del parral.

La producción de uva en la zona septentrional del País Vasco peninsular contaba con la problemática derivada de una climatología muy húmeda. Ya San Isidoro de Sevilla (627-630) señalaba "... allá donde la niebla o la humedad general (. ..) parecían hacer prohibitivo la existencia de la vid, allí el hombre consiguió crear el viñedo para el mejor vino". Para evitar problemas de botritis, las parras de los viñedos vascos tenían que estar elevadas del suelo; para ello se empleaban distintos tipos de tutores y sistemas de conducción: árboles (Herrera, 1513; San Martín, 1791), rodrigones, huesos de ballena (Humboldt, 1799), o se conducían por las paredes de los caseríos y granjas (Herrera, 1513). Este último tipo de guía se llevaba a cabo mediante piezas de madera incrustadas, largos clavos de forja o mediante perrotiak, es decir, canes o círculos de piedra integrados en la fachada principal desde su construcción (Santana, 2001). Estos últimos soportes son propios de casas de piedra de buena factura, erigidas en el siglo XVIII y obedeciendo al estilo barroco (Baeschlin, 1930).

El inglés John Moore, durante su visita en tiempos de la primera Guerra Carlista (1835-1840) a la zona, describía, según recogieron Sillaurren \& Santamaría (1978) que "... a los lados de la carretera que sale de Amurrio, y por los hermosos campos de los alrededores, hay un gran número de caseríos o pequeñas casas de campo con amplias techumbres de estilo suizo; las parras cubren las blancas paredes y dan sombra a las ventanas...". Por su parte, Becerro de Bengoa visitaba Ayala en 1880, y cerca de Menagarai hacía la siguiente descripción: "Por las huertas y a lo largo de las paredes, 
alargan las parras sus retorcidos brazos... y por la dilatada población de Luyando con sus baños y su viejo puente ojival, y la multitud de típicos caseríos adornados de parras".

La llegada de las enfermedades fúngicas del oídio y mildiu, así como de la filoxera, entre el último tercio del siglo XIX y principios del XX, hicieron que la superficie de viñedo en la zona del chacolí alavés se redujera de las 1.085 ha existentes con anterioridad a 1909 a únicamente 93 ha con posterioridad a dicha fecha, y todas ellas plantadas de vid americana (Junta Consultiva Agronómica, 1911). Por esos motivos se conservan varios topónimos que hacen referencia a antiguos viñedos desaparecidos.

Durante el citado periodo de principios del siglo XX, la reconstrucción del viñedo supuso la incorporación de portainjertos americanos y varios de sus híbridos con vinífera (híbridos productores directos) a la viticultura de este valle. Estos híbridos productores directos tenían la ventaja agronómica de no ser sensibles al mildiu, un grave problema sanitario que afecta con fuerte intensidad a las regiones vitícolas húmedas, como eran las zonas chacolineras situadas en el norte de Burgos, Cantabria y el País Vasco (Hidalgo et al., 2012). Lógicamente, las podas largas, que sólo buscaban producción, la falta de la madurez adecuada de la uva, extraída en buena parte de los citados híbridos, hacían que el chacolí fuera un vino de baja graduación y una alta acidez. Por esos motivos, su consumo era meramente local.

En 2003 nació la Denominación de Origen Chacolí de Álava / ArabakoTxakolina. En los nuevos viñedos, se eliminaron los híbridos productores directos y sólo se permiten viníferas de calidad, adaptadas a las condiciones climatológicas de la zona. Fundamentalmente, Hondarribi Zuri (Courbu), Hondarribi Beltza, Hondarribi Zuri Zerratia (Petit Courbu), Izkiriota (Gross Manseng) e Izkiriota Tipia (Petit Manseng) (Hidalgo \& Llano, 2007).

El conjunto de variedades blancas es muy similar al de la zona francesa de Jurançon. Cabe destacar que la variedad tinta Hondarribi Beltza fue ya citada por García de los Salmones (1914) en la zona chacolinera de Getaria (Gipuzkoa). En el departamento francés de los Pirineos Occidentales, se la suele conocer bajo la sinonimia de chacolí.

El objetivo del presente artículo es conocer la distribución de las poblaciones silvestres en la Cuadrilla de Ayala, así como sus principales características ampelográficas y sanitarias, para implementar medidas para su conservación. Dada la importante tradición de las parras adosadas al frontal de los caseríos desde el siglo XVIII, también se ha acometido la identificación de aquellos ejemplares de origen antiguo que aparecen en la fachada principal de varios caseríos de la Cuadrilla de Ayala (Figura 1). 


\section{Material y métodos}

Primero se llevó a cabo una prospección de parras en los bosques de ribera de la red fluvial del territorio, así como de las zonas coluviales de sus entornos, durante el periodo de floración, entre 20 de junio y 10 de julio del periodo 2012-2015. Tras comprobar el carácter dioico de las mismas, las coordenadas de las diferentes poblaciones fueron tomadas mediante un GPS. Asimismo, se determinaron las principales especies de lianas, árboles y arbustos de la flora acompañante utilizando las claves botánicas de Flora iberica (Castroviejo \& al., 1986-2017).

Para el estudio del estado sanitario de las raíces de los ejemplares silvestres, se realizaron catas para su observación hasta una profundidad máxima de $30 \mathrm{~cm}$. En la parte aérea, se observaron los posibles síntomas causados por especies parásitas tanto en sarmientos, hojas y racimos hasta unos $3 \mathrm{~m}$ de altura.

Las parras de los caseríos muestreados fueron seleccionadas tras recibir información sobre la posible antigüedad de las mismas por parte de sus dueños. Únicamente se tuvieron en cuenta aquellas que fueron plantadas hace más de 50 años.

Para la realización del estudio genético, tanto de las parras silvestres como de las seleccionadas en los caseríos, se tomaron en verano muestras de hojas jóvenes de pequeño tamaño, recién expandidas, para la extracción de su ADN. Dicho muestreo se continuó con la recogida de sarmientos con madera de uno y dos años, una vez agostados, que fueron llevados al laboratorio con el fin de inducir artificialmente su brotación y obtener nuevas hojas con las que complementar su estudio genético.

Las extracciones de ADN se llevaron a cabo utilizando el Plant DNeas y Kit de Qiagen, de acuerdo al protocolo del fabricante. Los ADNs se estudiaron empleando los 48 marcadores SNPs descritos con anterioridad (Cabezas et al., 2011), en el Centro Nacional de Genotipado (CeGen). Los resultados fueron analizados, comparando los genotipos obtenidos entre sí y con la base de datos disponible ICVV-SNP. Asimismo, en las vides silvestres se utilizaron los genotipos de SNP para estimar las similitudes genéticas en base a la frecuencia de alelos compartidos (Bowcock et al.1994).

\section{Resultados y discusión}

\section{Vides silvestres}

La localización de los diferentes núcleos poblacionales de vid silvestre encontrados se recogen en la Tabla 1 (Figura 1).

En total se han encontrado 8 poblaciones de vid silvestre, con un total de 57 parras, 37 masculinas y 20 femeninas, de las que se muestrearon 24 para análisis de ADN (Tablas 1 y 2). 


\begin{tabular}{|lccc|}
\hline Pueblo/municipio & Masculinas & Femeninas & $\begin{array}{c}\mathbf{N}^{\circ} \text { muestras } \\
\text { para ADN }\end{array}$ \\
\hline Amurrio & 8 & 6 & 7 \\
Saratxo (Amurrio) & 4 & 1 & 3 \\
Etxegoien (Ayala) & 5 & 3 & 5 \\
Olabezar (Ayala) & 3 & 1 & 2 \\
Zuhatza (Ayala) & 2 & 1 & 1 \\
Quejana (Ayala) & 6 & 4 & 2 \\
Respaldiza (Ayala) & 4 & 2 & 2 \\
Artziniega & 5 & 2 & 2 \\
\hline
\end{tabular}

Tabla 1.- Número de parras de cada sexo por población.

Table 1.- Number of vines of each sex by population.

La comparación de las muestras entre sí permitió encontrar dos coincidencias completas entre las muestras S13 y S14 por un lado y S19 y S20 por otro, para todos los SNPs comparados. Lo más probable es que se trate de las mismas plantas en cada caso, y han debido originarse por una multiplicación vegetativa espontánea de la planta original. Las plantas más cercanas genéticamente son S3 y S4, de Amurrio, con una similitud de 0,94, mientras que las más lejanas son S9 (Saratxo) y S14 (Etxegoien), con una similitud de 0,62 . La similitud promedio en los 22 genotipos únicos ha sido de 0,79 .

Se observa que, para todas las plantas menos las de Zuhatza (S18), existen genotipos cercanos en la base de datos ICVV-SNP, que muestran entre 4 y 10 diferencias con las estudiadas. En todos los casos esos genotipos cercanos corresponden a plantas silvestres de vid, mayoritariamente procedentes del País Vasco, seguidas por otras zonas del norte de la Península Ibérica, como Cantabria y Asturias. Estos resultados ponen de manifiesto la conocida reducción de variabilidad existente entre las plantas silvestres de vid en muchas partes de Europa, debido al bajo número de individuos que conforman sus poblaciones, que ha llevado a la desaparición de muchas de ellas, provocado fundamentalmente por la acción humana.

Las flores de las plantas masculinas son todas tipo I, de acuerdo con los descriptores de OIV (2009). Es decir, sin ningún desarrollo de gineceo. En general, las hojas adultas de las lianas femeninas son de mayor tamaño y menos lobuladas que las masculinas (Figura 2).

En cuanto a la vegetación acompañante, entre la que se encuentran varios tutores de las parras, las especies más frecuentes son: Acer campestre L., A. monspessulanum L., Alnus glutinosa (L.) Gaertn., Arbutusunedo L., Clematis vitalba L., Crataegus monogyna Jacq., Ficus carica L., Fraxinus angustifolia Vahl, Populusnigra L., Quercus 


\begin{tabular}{|c|c|c|c|c|}
\hline $\begin{array}{l}\text { Código } \\
\text { muestra }\end{array}$ & $\begin{array}{l}\text { Denominación } \\
\text { muestra }\end{array}$ & $\begin{array}{l}\text { Pueblo/ } \\
\text { municipio }\end{array}$ & Latitud N & Longitud $\mathrm{O}$ \\
\hline S1 & $\begin{array}{c}\text { Zona caserío Araneko y } \\
\text { circunvalación de Amurrio }\end{array}$ & Amurrio & $43^{\circ} 02^{\prime} 26,80^{\prime \prime}$ & $02^{\circ} 59^{\prime} 24,70^{\prime \prime}$ \\
\hline S2 & $\begin{array}{l}\text { Zona caserío Araneko y } \\
\text { circunvalación de Amurrio }\end{array}$ & Amurrio & $43^{\circ} 02^{\prime} 26,80^{\prime \prime}$ & $02^{\circ} 59^{\prime} 24,70^{\prime \prime}$ \\
\hline S3 & $\begin{array}{l}\text { Zona caserío Araneko y } \\
\text { circunvalación de Amurrio }\end{array}$ & Amurrio & $43^{\circ} 02^{\prime} 26,00^{\prime \prime}$ & $02^{\circ} 59^{\prime} 24,80^{\prime \prime}$ \\
\hline S4 & $\begin{array}{c}\text { Zona caserío Araneko y } \\
\text { circunvalación de Amurrio }\end{array}$ & Amurrio & $43^{\circ} 02^{\prime} 26,40^{\prime \prime}$ & $02^{\circ} 59^{\prime} 25,00^{\prime \prime}$ \\
\hline S5 & $\begin{array}{c}\text { Zona caserío Araneko y } \\
\text { circunvalación de Amurrio }\end{array}$ & Amurrio & $43^{\circ} 02^{\prime} 28,90^{\prime \prime}$ & $02^{\circ} 59^{\prime} 25,10^{\prime \prime}$ \\
\hline S6 & $\begin{array}{l}\text { Zona caserío Araneko y } \\
\text { circunvalación de Amurrio }\end{array}$ & Amurrio & $43^{\circ} 02^{\prime} 27,30^{\prime \prime}$ & $02^{\circ} 59^{\prime} 25,30^{\prime \prime}$ \\
\hline S7 & $\begin{array}{l}\text { Zona caserío Araneko y } \\
\text { circunvalación de Amurrio }\end{array}$ & Amurrio & $43^{\circ} 02^{\prime} 29,60^{\prime \prime}$ & $02^{\circ} 59^{\prime} 23,90^{\prime \prime}$ \\
\hline S8 & Entre C-625 y río Nervión & Saratxo, Amurrio & $43^{\circ} 01^{\prime} 28,00^{\prime \prime}$ & $03^{\circ} 00^{\prime} 34,40^{\prime \prime}$ \\
\hline S9 & Entre C-625 y río Nervión & Saratxo, Amurrio & $43^{\circ} 01^{\prime} 30,70^{\prime \prime}$ & $03^{\circ} 00^{\prime} 33,70^{\prime \prime}$ \\
\hline S10 & $\begin{array}{l}\text { Lado izquierdo C-625 } \\
\text { sentido Orduña }\end{array}$ & Saratxo, Amurrio & $43^{\circ} 01^{\prime} 30,40^{\prime \prime}$ & $03^{\circ} 00^{\prime} 33,20^{\prime \prime}$ \\
\hline S11 & $\begin{array}{l}\text { San Esteban junto } \\
\text { arroyo Larruzabal }\end{array}$ & Etxegoien, Ayala & $43^{\circ} 02^{\prime} 39,60^{\prime \prime}$ & $03^{\circ} 00^{\prime} 53,30^{\prime \prime}$ \\
\hline S12 & $\begin{array}{l}\text { San Esteban junto } \\
\text { arroyo Larruzabal }\end{array}$ & Etxegoien, Ayala & $43^{\circ} 02^{\prime} 42,00^{\prime \prime}$ & $03^{\circ} 00^{\prime} 55,20^{\prime \prime}$ \\
\hline S13 & $\begin{array}{l}\text { San Esteban junto } \\
\text { arroyo Larruzabal }\end{array}$ & Etxegoien, Ayala & $43^{\circ} 02^{\prime} 41,80^{\prime \prime}$ & $03^{\circ} 00^{\prime} 59,20^{\prime \prime}$ \\
\hline S14 & $\begin{array}{l}\text { San Esteban junto } \\
\text { arroyo Larruzabal }\end{array}$ & Etxegoien, Ayala & $43^{\circ} 02^{\prime} 41,00^{\prime \prime}$ & $03^{\circ} 00^{\prime} 59,90^{\prime \prime}$ \\
\hline S15 & $\begin{array}{l}\text { San Esteban junto } \\
\text { arroyo Larruzabal }\end{array}$ & Etxegoien, Ayala & $43^{\circ} 02^{\prime} 41,00^{\prime \prime}$ & $03^{\circ} 01^{\prime} 01,60^{\prime \prime}$ \\
\hline S16 & $\begin{array}{c}\text { Entre caserío Arriaga } \\
\text { y bifurcación }\end{array}$ & Olabezar, Ayala & $43^{\circ} 03^{\prime} 13,90^{\prime \prime}$ & $03^{\circ} 01^{\prime} 03,20 "$ \\
\hline S17 & $\begin{array}{c}\text { Entre caserío Arriaga } \\
\text { y bifurcación }\end{array}$ & Olabezar, Ayala & $43^{\circ} 03^{\prime} 13,90^{\prime \prime}$ & $03^{\circ} 01^{\prime} 04,30^{\prime \prime}$ \\
\hline S18 & $\begin{array}{c}\text { Puente sobre río Itzalde } \\
\text { a barrio Undio }\end{array}$ & Zuhatza, Ayala & $43^{\circ} 07^{\prime} 42,10^{\prime \prime}$ & $03^{\circ} 02^{\prime} 06,70^{\prime \prime}$ \\
\hline S19 & La Serna, junto al río Itzalde & Quejana, Ayala & $43^{\circ} 04^{\prime} 37,90^{\prime \prime}$ & $03^{\circ} 04^{\prime} 21,80^{\prime \prime}$ \\
\hline S20 & La Serna, junto al río Itzalde & Quejana, Ayala & $43^{\circ} 04^{\prime} 38,30^{\prime \prime}$ & $03^{\circ} 04^{\prime} 21,90^{\prime \prime}$ \\
\hline S21 & La Lastra (sobre A-624) & Respaldiza, Ayala & $43^{\circ} 05^{\prime} 03,70^{\prime \prime}$ & $03^{\circ} 03^{\prime} 08,30^{\prime \prime}$ \\
\hline S22 & La Lastra (sobre A-624) & Respaldiza, Ayala & $43^{\circ} 05^{\prime} 08,90^{\prime \prime}$ & $03^{\circ} 03^{\prime} 17,60^{\prime \prime}$ \\
\hline S23 & El Refugio & Artziniega & $43^{\circ} 06^{\prime} 49,70^{\prime \prime}$ & $03^{\circ} 09^{\prime} 08,40^{\prime \prime}$ \\
\hline$\$ 24$ & El Refugio & Artziniega & $43^{\circ} 06^{\prime} 48,40^{\prime \prime}$ & $03^{\circ} 09^{\prime} 11,10^{\prime \prime}$ \\
\hline
\end{tabular}

Tabla 2.- Denominación y localización de las plantas de vid silvestre prospectadas para el análisis de ADN. Table 2.- Denomination and location of wild grapevine plants prospected for DNA analysis. 
Hidalgo et al.

humilis Mill., Quercus robur L., Rubus ulmifolius Schott, Salix alba L., Salixtriandra L., Sambucus nigra L. y Tilia cordata Mill.

Referente al estado sanitario de las raíces, cabe destacar la ausencia de síntomas atribuibles a la fase radicícola de la filoxera (Daktulosphaira vitifoliae Fitch), alimentándose de los pelos absorbentes, así como de nódulos provocados por nematodos.

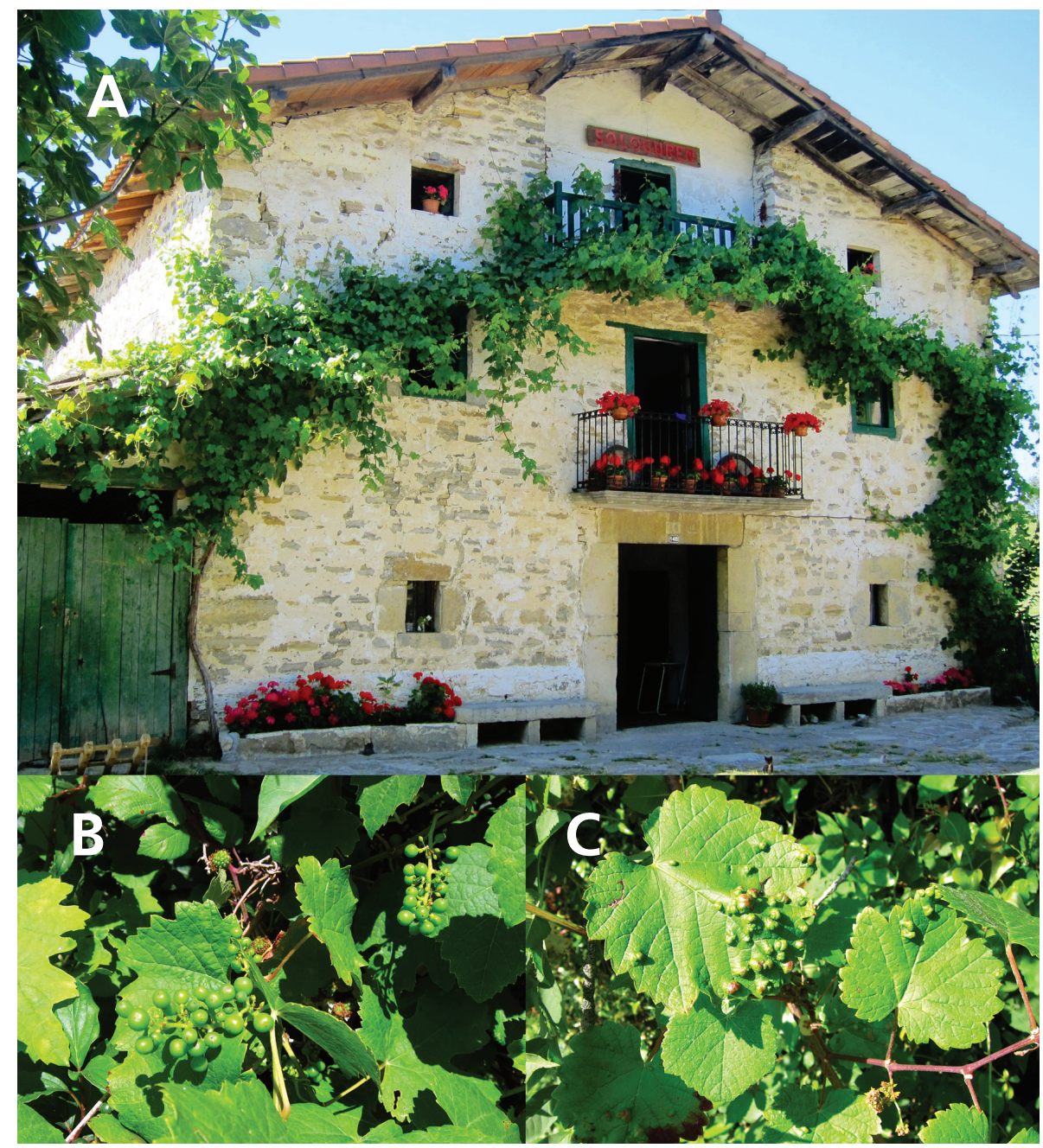

Fig 2.- A) Caserío Sologuren en Quejana, con parra en su fachada principal. (B) Ejemplar femenino con bayas en tamaño guisante, Amurrio. (C) Parra con síntomas de erinosis, Santa Coloma (Artziniega).

Fig 2.- Map of the situation of the Land of Ayala in the province of Álava and in Spain. Location of the samples of cultivated vines collected on the facades of the farmhouses of Cuadrilla de Ayala, as well as of wild vines, for the present study. 
Tampoco se ha observado la presencia de micelios blanquecinos de hongos asociados a la podredumbre. En particular, llama la atención que no se hayan detectado micelios de Armillaria mellea (Vahl.) Kumm., que, sin embargo, son bastante frecuentes en los chopos que forman parte de la vegetación de ribera acompañante de las parras y que, en ocasiones, sirven como tutor a las mismas.

La ausencia de infestación por filoxera y de nódulos causados por nematodos parece deberse a las condiciones especiales de contenido en agua en el perfil de los fluvisoles sobre los que se asientan la mayor parte de estas poblaciones, que sufren dilatados periodos de encharcamiento cada año. Cabe apuntar que las raíces de las parras silvestres fuera de su medio natural sí son sensibles a la fase radicícola de la filoxera. En ese sentido, existe un precedente curioso en Francia, narrado por Camile Saint Pierre, en el que se recoge que los sarmientos de los ejemplares silvestres se llegaron a emplear como portainjerto en algunos viñedos reconstituidos para intentar remediar el problema causado por el citado áfido. Al cabo de cierto tiempo, comenzaron los síntomas de infestación con la consiguiente muerte de las cepas (De La Branchére, 1876).

La presencia de falsas agallas causadas por el ácaro de la erinosis, Colomerus vitis (Pagenstecher) (Acari, Eriophyidae), sobre hojas de las parras silvestres se ha detectado en la totalidad de los ejemplares muestreados (Figura 2). Algo inferior es la correspondiente a las zonas decoloradas por efecto de la alimentación de las colonias del agente responsable de la acariosis, Calepitrimerus vitis (Nalepa) (Acari, Eriophyidae).

Los síntomas causados por oídio, Erysiphe necator (Schwein.) Burriel, y mildiu, Plasmopara viticola (Berlease $\&$ de Toni) son palpables tanto en los órganos vegetativos como en los racimos, desde su cuajado hasta el estado de maduración de las bayas, a finales de octubre.

Los datos expuestos coinciden ampliamente con los encontrados sobre un amplio conjunto de poblaciones europeas de vid silvestre que han sido estudiadas desde Portugal hasta Hungría (Ocete et al., 2011b). Ello parece indicar que ambos ácaros eriófidos monófagos pasaron de los ejemplares silvestres a los cultivados durante el proceso de domesticación de la vid. En sentido opuesto, los citados hongos, importados de Norteamérica durante el siglo XIX sobre ejemplares de vid americana, han pasado de los viñedos a las poblaciones silvestres de la zona.

Los pequeños núcleos dispersos relacionados en este trabajo, así como los que aún se conservan en los Valles de Mena y Angulo (Burgos) y en Las Encartaciones (Bizkaia) parece ser un reflejo de mayores poblaciones que se asentaban en esta zona geográfica con fuerte influencia atlántica, y que fueron secularmente aprovechadas para la producción de remedios medicinales (Bustamante, 1971). Las explotaciones de los bosques de ribera, para uso hortícola y forestal, así como el impacto causado por las especies fúngicas norteamericanas fueron, probablemente, las causas principales de 
la rarificación de este taxón, como ha ocurrido en otras regiones europeas (Arnold, 2002). A ello, hay que añadir la construcción de las carreteras y su mantenimiento, principalmente el desbroce de las cunetas. En relación con este último factor negativo, hay que señalar la pérdida de tres ejemplares en la zona de Saratxo, junto al río Nervión, durante la realización de este trabajo.

Convendría solicitar al Gobierno Vasco una ley para la protección específica in situ de este taxón amenazado. Por otra parte, habría que salvaguardar urgentemente este material en bancos de germoplasma, como se hizo con las parras de Bizkaia, que se conservan en la Estación de Fruticultura de Zalla (Bizkaia). También en reservas, como la establecida en torno al Lago de Arreo (Cuadrilla de Añana) por el Servicio de Biodiversidad de la Diputación Foral de Álava.

\section{Vides de caseríos}

Se muestrearon 35 parras antiguas de caseríos, así como 6 plantas que crecían probablemente asilvestradas, con características ampelográficas atípicas de las realmente silvestres, a nivel de morfología foliar y sexo (Figuras 1 y 2 y apéndice final).

El análisis de marcadores de ADN y posterior comparación con la base de datos ICVVSNP ha permitido identificar la mayoría de muestras. Graciano es la variedad que ha aparecido con mayor frecuencia (9 muestras), seguida de De José Blanco (o Catalán Blanco, 6 muestras) y Moscatel de Alejandría (4 muestras). Resulta llamativo que aparecen varios híbridos productores directos: Baco Noir, De José Blanco, Duc Petit y Vivarais. Se trata de híbridos interespecíficos que actualmente no se pueden utilizar en España para elaborar vino, dado que sólo se permite el uso de variedades de Vitis vinifera (Ley 24/2003, de 10 de julio, de la Viña y del Vino, BOE de 11 julio 2003). Entre las plantas asilvestradas (cuyo código de muestra comienza por 'E') hay 4 que genéticamente están más próximas a las silvestres, por lo que podrían tratarse de silvestres o descendientes de hibridaciones espontáneas entre plantas silvestres y cultivadas. Pero se incluyen en este grupo por su proximidad a los caseríos. Asimismo también se ha podido identificar una planta que procede de una semilla de Graciano producida por autofecundación (C2A).

Seguidamente se recoge brevemente información de interés sobre las 15 variedades identificadas en el presente artículo, parte de la cual se ha tomado del Catálogo Internacional de Variedades de Vid (www.vivc.de).

Courbu Blanc: Conocida en el País Vasco como Ondarribi Zuri, es una variedad de origen desconocido, que cita por primera vez García de los Salmones (1914) en la zona de Guipúzcoa. En la actualidad su cultivo se localiza exclusivamente en el País Vasco, dónde está admitida en las tres D.O. Chacolí. En Francia se cultiva de forma 
muy minoritaria bajo el nombre de Courbu Blanc. Se trata de una variedad de baja producción con racimos pequeños y alados de compacidad alta. Las uvas son pequeñas y esféricas, de color amarillo dorado con pecas marrones. Sus vinos son ácidos y poco alcohólicos con aromas cítricos, vegetales y florales.

Folle Blanche: La variedad Folle Blanche es una variedad de origen francés, posiblemente de la región de Charente en el sudoeste de Francia dónde está citada desde el siglo XVII (Cabello et al., 2011). En España se la conoce con el nombre de Matza Zuri y su cultivo está casi exclusivamente limitado al País Vasco, en donde está autorizada en la D.O. Bizkaiko Txakolina-Chacolí de Bizkaia. Esta variedad es de producción intermedia y presenta racimos muy compactos de tamaño entre pequeño y mediano y forma cilíndrica con un ala. La uva es de color verde amarillo, tamaño medio y forma acuminada. Los vinos son ácidos y poco alcohólicos con aromas a manzana verde.

Graciano: Según Manso de Zuñiga (1905), esta variedad podría ser originaria de La Rioja. Su cultivo se extiende desde muy antiguo a muchas regiones vitivinícolas españolas, llegando también a Portugal con el nombre de Tinta Miuda, a Francia con el nombre de Morrastel y a Italia con el nombre de Cagniulari. En Andalucía se la conoce también con el nombre de Tintilla de Rota. La cita más antigua corresponde a la denominación de Tintilla (Clemente y Rubio, 1807); es Abela (1885) el que la cita como Graciano en viñedos de Álava y Burgos y como Graciana en La Rioja y Bizkaia. Con la Tintilla de Rota (Cádiz) se producían los vinos dulces que se exportaban a Irlanda para su utilización como vino de misa (Maldonado, 2004). Posteriormente ha sido citada en múltiples tratados de ampelografía y viticultura por diversos autores (véase Cabello et al., 2011). Graciano es una variedad de vinificación que produce racimos medianos y compactos de forma cilindro-cónica corta. Las uvas son de tamaño medio, esféricas, de color azul negro y sabor neutro. Es de brotación y maduración tardías y alta producción. Sus vinos suelen ser de graduación media-alta y acidez elevada.

Mollar Cano: La variedad Mollar Cano, también conocida Negramoll, entre otras sinonimias, es una variedad antigua de la Península Ibérica que es citada por primera vez con el nombre de Mollar por Valcárcel (1791). Cabello et al., (2011) proponen su origen en el suroeste de la Península Ibérica, concretamente en las provincias de Huelva, Cádiz y Sevilla y en el sur de Portugal. Desde allí se habría llevado a las Islas Canarias donde en la actualidad está autorizada en la mayor parte de sus Denominaciones de Origen. Esta variedad fue llevada a América posiblemente a principios del siglo XVI y generó allí algunas de las variedades denominadas criollas (Milla-Tapia et al., 2007). Mollar Cano es una variedad de producción alta que presenta racimos grandes y de compacidad media con forma cónica alargada. Las uvas son grandes y esféricas, con 
un color variable entre el rosado y el violeta oscuro. Produce vinos de grado alcohólico medio-alto pero de acidez y color escasos. Con ella se elaboraban las famosas Mistelas de Los Palacios y Villafranca (Sevilla) que se vendían para incrementar el contenido en azúcar de determinadas elaboraciones del Marco del Jerez.

Moscatel de Alejandría: Podría ser una de las variedades más antiguas que todavía se cultivan. En la actualidad está extendida por todos los países mediterráneos con distintos nombres que hacen referencia a su intenso aroma moscatel. En España se la conoce con el nombre de Moscatel de Alejandría, Moscatel de Málaga o Moscatel de grano gordo para distinguirla de uno de sus progenitores, Moscatel de grano menudo. Las variedades de aroma moscatel están citadas ya en los tratados de agricultura más antiguos (Columela, siglo I) y posteriormente por Herrera (1513) aunque las descripciones no permiten discernir si se refieren a Moscatel de Alejandría, a Moscatel de grano menudo o a otras variedades con este tipo de aroma. Moscatel de Alejandría es una variedad de producción media-alta que presenta racimos de tamaño mediogrande, sueltos y alados con forma piramidal. La uva es de color verde amarillo, tamaño grande y forma elíptica larga con sabor moscatel. Esta variedad se utiliza como uva de mesa, de vinificación y también para la producción de pasas. Los vinos de Moscatel de Alejandría son de graduación media y acidez baja.

Moscatel de Estambul: Variedad posiblemente utilizada para uva de mesa, cultivada en Israel y en Túnez (Galet, 2000) y presente también en la Península Ibérica dónde ha sido localizada en la región de Castilla La Mancha (Mena et al., 2014). Los datos de pedigrí avalan está distribución dado que desciende de la hibridación de la variedad Moscatel de Alejandría y la variedad ibérica Beba o Valencí Blanco distribuidas ambas en esas regiones. Es una variedad productiva con racimos grandes, alados y troncocónicos. Las uvas son blancas, grandes y ovoides con pulpa un poco fibrosa y sabor intenso a moscatel (Galet, 2000).

Moscatel de Hamburgo: Es una variedad de uva de mesa seleccionada en Gran Bretaña, hacia 1850, por el mejorador Robert Snow a partir de un cruzamiento entre las variedades Schiava Grossa y Moscatel de Alejandría. De producción media-alta, posee racimos alados cónicos de tamaño y compacidad media. Las uvas, de color rojo-violeta oscuro, son grandes y elípticas y de sabor moscatel. El potencial enológico es bajo, produciendo vinos de grado alcohólico medio y acidez media-baja.

Naparo: Variedad de uva de mesa de origen ibérico también conocida como Alicante Rosa. Podría tratarse de una variedad muy antigua dado que es una de las pocas varie- 
dades femeninas que se conocen en la Península Ibérica. De producción media, genera racimos alados cónicos de tamaño medio y compacidad baja. Las uvas son grandes y elípticas de color blanco rosado y sabor neutro.

Palomino fino: La uva palomina es citada inicialmente por Herrera (1513). Posteriormente Clemente y Rubio (1807) se refiere a ella como Listán Blanco, sinonimia que todavía se utiliza en algunas zonas de cultivo. Posiblemente se trata de una variedad antigua originaria del sudoeste de la Península Ibérica que junto a otras variedades se extendieron por el centro y norte por la Ruta de la Plata (Cabello et al., 2011). En la actualidad está distribuida ampliamente por Andalucía (Marco del Jerez), Canarias y Galicia, y también se localiza en Castilla y León. En el norte de Portugal recibe el nombre de Seminario. De producción elevada, presenta racimos alados de tamaño medio-grande, compacidad alta y forma cónico-piramidal. Las uvas son grandes y esféricas de color amarillo dorado y sabor neutro. Sus vinos son de grado alcohólico medio y baja acidez. Una vez encabezados con alcohol vínico se destinan a la producción de Vino Fino, Amontillado, Oloroso y otras elaboraciones del Marco del Jerez. Asimismo, esta variedad es la materia prima de la Manzanilla de Sanlúcar de Barrameda, en Cádiz.

Sená o Señá: Es citada por primera vez en Bizkaia por Abela (1885), mientras que García de los Salmones (1914) la cita tanto en Álava como en Bizkaia. En la actualidad se cultiva de forma muy reducida en algunas localidades de ambas provincias (Cabello et al., 2011). Es una variedad de producción baja que presenta racimos con un ala, de tamaño grande y forma cónica y de baja compacidad. Las uvas son grandes y esféricas de color azul-negro. Los vinos de esta variedad son de acidez y grado alcohólico medios (Cabello et al., 2011). Con esta variedad se obtienen los chacolís con color Ojo de gallo en Las Encartaciones (Bizkaia) y en Valle de Mena (Burgos).

Tinto Velasco: El origen de esta variedad, también conocida como Velasco o Blasco, es desconocido, aunque según Cabello et al., (2011) podría proceder de Aragón o Navarra. Clemente y Rubio (1807), cita por primera vez una variedad denominada Blasca y cultivada en Baza (Granada) y comenta que podría ser la misma que llaman Velasco en Tembleque (Toledo) y Blasco en Titaguas y otros pueblos de Valencia. Posteriormente ha sido citada en múltiples tratados de ampelografía y viticultura por diversos autores (véase Cabello et al., 2011). De producción media alta, da racimos grandes y alados de forma cónica y compacidad alta. Las uvas son grandes y elípticas de color azul negro y sabor neutro. Sus vinos son de graduación media y acidez alta. 
Baco noir: Conocido en España como Baco negro o Baco 1, es un híbrido productor directo seleccionado en Francia en 1902 por el mejorador François Baco a partir de un cruzamiento entre la variedad Folle Blanche, descrita más arriba, y la accesión Riparia Gran Glabre de Vitis riparia. Utilizado para la elaboración de vino, se caracteriza por una maduración precoz y una producción media. Presenta racimos cilíndricos de tamaño medio y compacidad alta, y uvas pequeñas y esféricas de color azul negro y aromas herbáceos. Sus vinos son de graduación media y acidez elevada.

De José Blanco: Esta variedad, ampliamente conocida en el norte de la Península Ibérica como Catalán Blanco, es un híbrido productor directo de origen desconocido. Produce racimos cilíndricos, alados, de compacidad media (Loureiro et al., 2011). Las uvas son blancas y aromáticas y se utilizan para elaborar un aguardiente muy aromático.

Duc Petit: También conocido como Seibel 156, Duc Petit es un híbrido productor directo seleccionado en Francia en 1886, por el mejorador Albert Seibel a partir de un cruzamiento entre un híbrido interespecífico (Vitis rupestris $\times \mathrm{V}$. lincecumii) y una línea de $V$. vinifera. Utilizado como portainjertos, pero también para la elaboración de vino y como uva de mesa, se caracteriza por ser muy productivo y presentar racimos de forma cilíndrica, tamaño pequeño y compacidad media. Las uvas son pequeñas y esféricas de color azul negro. Sus vinos suelen ser de graduación alta y acidez elevada.

Vivarais: También conocida como Seibel 2003, es un híbrido productor directo seleccionado en Francia por el mejorador Albert Seibel a partir de un cruzamiento entre Munson ( $V$. lincecumii $x V$. rupestris) y Herbemont Dáurelles 1 [ $(V$. aestivalis $x$ $V$. cinerea) polinización abierta]. Utilizado para la elaboración de vino, se caracteriza por una maduración media y una producción media. Presenta racimos cilíndricos y pequeños de compacidad media y uvas elípticas de tamaño medio a grande, de color azul negro y sabor neutro. Sus vinos son de graduación media acidez alta.

\section{Agradecimientos}

A Joseba Carreras de Bergareche, Jefe de Sección de Biodiversidad de la Dirección de Medio Ambiente y Urbanismo de la Diputación Foral de Álava, que ha ayudado económicamente en la realización de los análisis de las parras silvestres. 


\section{Bibliografía}

Abela, E. 1885. El libro del viticultor. Editorial Maxtor. Madrid.

Arnold, C. 2002. Ecologie de la vigne sauvage, Vitis vinifera L. ssp sylvestris (Gmelin) Hegi, dans les forêts alluviales et colluviales d'Europe. Geobotánica Helvética. Univ. Neuchâtel. Suiza.

Arroyo-García, R., Ruiz-García, L., Bolling, L., Ocete, R., López, M.A., Arnold, C, Ergul, A., Söylemezo, G., Uzun, I., Cabello,F., Ibañez, J., Aradhya, M.K., Atanassov, A., Atanassov, I., Balint, S., Cenis, J.L., Costantini, L., Gorislavets, S., Grando, M.S., Klein, B.Y., McGovern, P. E., Merdinoglu, D., Pejic, I., Pelsy, F., Primikirios, N., Risovannaya, V., Roubelakis-Angelakis, K.A., Snoussi, H., Sotiri, P., Tamhankar, S., This, P., Troshin, L., Malpica, J.M., Lefort, F., Martínez-Zapater, J.M. 2006. Multiple origins of cultivated grapevine (Vitis vinifera L. ssp. sativa) based on chloroplast DNA polymorphisms. Mol. Ecol. 15(12): 3707-3714.

Baeschlin, A. 1930. La arquitectura del caserío vasco. Editorial Canosa. Barcelona.

Bowcock, A.M., Ruiz-Linares, A., Tomfohrde, J., Minch, E., Kidd, J. R., Cavalli-Sforza, L.L. 1994. High resolution of human evolutionary trees with polymorphic microsatellites. Nature 368: 455-7

Bustamante, J. 1971. La Tierra y los Valles de Mena. Salustegui Editores. Bilbao.

Cabello, F., Ortiz, J.M., Muñoz-Organero, G., Rodríguez-Torres, I., Benito, A., Rubio, C., García-Muñoz, S., Sáiz, R. 2011. Variedades de Vid en España. Comunidad de Madrid \& Editorial Agrícola. Madrid.

Cabezas, J.A., Ibáñez, J., Lijavetzky, D., Vélez, M.D., Bravo, G., Rodríguez, V., Carreño, I., Jermakow, A.M., Carreño, J., Ruiz-García, L., Thomas, M.R.Martínez-Zapater, J.M.2011.A 48 snp set for grapevine cultivar identification. BMC Plant Biology 11: 153. Doi: 10.1186/1471-2229-11-153.

Castroviejo, S. 1986-2017. Flora ibérica I-XVI (II), XVII, XVIII, XX, XXI. Real Jardín Botánico, CSIC. Madrid.

Clemente y Rubio, S.R. 1807. Ensayo sobre las variedades de la vid común que vegetan en Andalucía. Junta de Andalucía. Consejería de Agricultura y Pesca. Sevilla.

Chilashvili, L. 2004. The vine, Wine and Georgians. Authors of the idea. L. Gachechiladze, T. Kandelaki Tbilisi (en georgiano).

De la Blanchere, H. 1876. Les ravageurs des Vergers et des Vignes. J. Rothschild, Editeur. París.

Galet, P. 2000. Dictionnaire Encyclopédique des Cépages. Hachette. Paris.

García de los Salmones, N. 1914. Memoria General de las Sesiones del Congreso y Ponencias presentadas. Imprenta provincial. Pamplona.

Guinea, E. 1949. Bizkaia y su paisaje vegetal. Junta de Cultura de Bizkaia. Bilbao.

Herrera, G. Alonsode.1513. Obra de Agricultura copilada de diversos autores. Editor Arnao Guillén de Brocar. Alcalá de Henares. 
Hidalgo, J., Llano, J.M. 2007. Chacolí de Álava. Ed. J.M. Llano. Bilbao.

Hidalgo, J., Sáenz de Buruaga, T., Ocete, R. 2012. Vid cultivada y silvestre en el territorio de la antigua diócesis de Valpuesta (Álava, Burgos y Cantabria, España): un acercamiento a la historia del vino chacolí. Estudios Avanzados 18: 101-112. Universidad de Santiago de Chile. Disponible en: http://www.revistas.usach.cl/ojs/index.php/ideas/article/viewFile/1082/1020

Humboldt, W.1975. Los vascos. Apuntaciones sobre un viaje por el País Vasco en primavera del año1801. Auñamendi. San Sebastián.

Iriarte-Chiapusso, M.J., Gómez-Orellana, L., Muñoz-Sobrino, C., Ramil-Rego, P., Arrizabalaga, A. 2005. La dinámica de la vegetación en el NW peninsular durante la transición del Paleolítico Medio al Paleolítico Superior. En: Neandertales cantábricos, estado de la cuestión. R. Montes, J.A. Lasheras (Eds.): 231-253. Monografías del Museo de Altamira 20. Ministerio de Cultura. Madrid.

Iriarte-Chiapusso, M.J., Muñoz, C., Gómez-Orellana, L., Ramil-Rego, P. 2006. Dinámica del paisaje de la Reserva de la Biosfera del Urdaibai durante el Holoceno. Actas del III Congreso Español de Biogeografía: 113-117. Servicio de Publicaciones del Gobierno Vasco. Reserva de la Biosfera de Urdaibai. Álava.

Junta Consultiva Agronómica, 1911. La invasión filoxérica en España y estado en 1909 de la reconstrucción del viñedo. Ministerio de Fomento. Dirección General de Agricultura Minas y Montes. Madrid, M.G. Hernández.

Loureiro, M.D., Moreno-Sanz, P., Suárez, B. 2011. Variedades de vid de Asturias. SERIDA, Villaviciosa, Asturias.

Maldonado, J. 2004. El célebre Tintilla de Rota (siglos XVIII-XX). Fundación Alcalde Zoilo RuizMateos. Rota, Cádiz.

Manso de Zúñiga, V.C. 1905. Memoria anual. Estación Enológica de Haro. La Rioja.

Mena, A., Martínez, J., Fernández-González, M. 2014. Recovery, identification and relationships by microsatellite analysis of ancient grapevine cultivars from Castilla-La Mancha: the largest wine growing region in the world. Genetic Resources and Crop Evolution 61, 625-637. DOI: 10.1007/s10722-013-0064-3.

Milla-Tapia, A., Cabezas, J.A., Cabello, F., Lacombe, T., Martínez-Zapater, J.M., Hinrichsen, P.,Cervera, M.T., 2007. Determining the Spanish origin of representative ancient American grapevine varieties. Am. J. Enology and Vitic. 58: 242-251.

Morales, R., Ocete, R.2015. Vitis L. En: Flora Ibérica IX.F. Muñoz Garmendia, C. Navarro, A. Quintanar, A. Buira: 58-62.

Ocete, R. López, M.A., Gallardo, A., Arnold, C., Pérez, M.A. Rubio, I.M. 2004. Euskalherriko eta Inguruetako Basamahatsondoa: ekologia, kokapena eta arriskuak. Servicio Central de Publicaciones del Gobierno Vasco. Vitoria-Gasteiz.

Ocete, R., Muñoz-Organero, G., López, M.A., Pérez, M.A., Benito, A., Cabello, F., Valle, J.M. 2011a. Environmental, sanitary and ampelographic characterization of wild grapevine in western Pyrenees (Spain, France). Journal International des Sciences de La Vigne et du Vin, 45: 1-12. 
Ocete, R., Arnold, C., Failla, O., Lovicu, G., Biagini, B., Imazio, S., Lara, M., Maghradze, D., López, M.A. 2011b. Considerations on the European wild grapevine (Vitis vinifera L. ssp. Sylvestris (Gmelin) Hegi) and Phylloxera infestation. Vitis 50(2): 97-98.

OIV (Organización Internacional de la Viña y el Vino). 2009. Descriptor list for grape varieties and Vitis species. A Dendon. Paris.

Pérez Soler, M.D. 1970. Cartulario de Valpuesta. Editorial Anubar. Valencia.

San Martín Burgoa, A. 1791. El Labrador Vascongado, o antiguo agricultor español. Demonstración de las mejoras de que es susceptible la Agricultura en las Provincias Vascongadas, y de las grandes ventajas que se podrían lograr en todo el Reyno observando las reglas de la antigua labranza. Imprenta de Don Benito Cano. Madrid.

Santana Ezquerra, A., Larrañaga, J.A., Loinaz, J.L. Zulueta, A. 2001. La arquitectura del caserío de Euskal Herria. Historia y tipología, Vol. II. Servicio Central de Publicaciones del Gobierno Vasco. Vitoria-Gasteiz.

Sillaurren, R.M., Santamaría, J.M. 1978. Viajeros ingleses del siglo XIX. Luis de Ajuria. Vitoria.

Ubieto Arteta, A. 1976. Cartulario de San Millán de la Cogolla (759-1076). Instituto de Estudios Riojanos. Editorial Anubar. Valencia.

Valcárcel, J.A. 1791. Agricultura general y gobierno de la Casa de Campo. Joseph Estevan Dolz. Valencia.

Zapata Peña, L. 2002. Origen de la agricultura en el País Vasco y transformaciones en el paisaje: Análisis de restos vegetales arqueológicos. Kobie, Anejo 4. Bilbao. 
Hidalgo et al.

Apéndice. Localización e identificación genética de las plantas muestreadas en caseríos y posibles asilvestradas. Appendix. Location and genetic identification of the vines sampled in farmhouses and of possible feral vines.

\begin{tabular}{|c|c|c|c|c|c|}
\hline $\begin{array}{l}\text { Código } \\
\text { muestra }\end{array}$ & $\begin{array}{l}\text { Denominación } \\
\text { muestra }\end{array}$ & $\begin{array}{l}\text { Pueblo/ } \\
\text { municipio }\end{array}$ & Latitud N & Longitud O & Identificación \\
\hline C1 & $\begin{array}{c}\text { Caserío } n^{\circ} 6 \\
\text { (junto a C-624) }\end{array}$ & $\begin{array}{l}\text { Barrio la Cadena, } \\
\text { Olabezar (Ayala) }\end{array}$ & $43^{\circ} 3 ' 22,74 " \mathrm{~N}$ & $3^{\circ} 01^{\prime} 11,96 " 0$ & Graciano \\
\hline$C 2 \mathrm{~A}$ & $\begin{array}{c}\text { Caserío } n^{\circ} 5 \\
\text { (fachada principal) }\end{array}$ & $\begin{array}{l}\text { Barrio la Cadena, } \\
\text { Olabezar (Ayala) }\end{array}$ & $43^{\circ} 3^{\prime} 22,58^{\prime \prime} \mathrm{N}$ & $3^{\circ} 01^{\prime} 10,02^{\prime \prime} 0$ & $\begin{array}{l}\text { Semilla } \\
\text { de Graciano }\end{array}$ \\
\hline$C 2 B$ & $\begin{array}{c}\text { Caserío } n^{\circ} 5 \\
\text { (fachada lateral) }\end{array}$ & $\begin{array}{l}\text { Barrio la Cadena, } \\
\text { Olabezar (Ayala) }\end{array}$ & $43^{\circ} 3^{\prime} 22,45^{\prime \prime N}$ & $3^{\circ} 01^{\prime} 09,43^{\prime \prime} 0$ & Graciano \\
\hline C3A & $\begin{array}{l}\text { Casa del cura } \\
\text { Undio Arriba }\end{array}$ & $\begin{array}{l}\text { Bo Undio Arriba, } \\
\text { Zuhatza (Ayala) }\end{array}$ & $43^{\circ} 7^{\prime} 40,80^{\prime \prime N}$ & $3^{\circ} 01 ' 22,71^{\prime \prime} 0$ & $\begin{array}{l}\text { Moscatel de } \\
\text { Alejandría }\end{array}$ \\
\hline C $3 B$ & $\begin{array}{l}\text { Caserío Undio } \\
\text { Arriba } n^{\circ} 63\end{array}$ & $\begin{array}{l}\text { Bo Undio Arriba, } \\
\text { Zuhatza (Ayala) }\end{array}$ & $43^{\circ} 7^{\prime} 40,52 " \mathrm{~N}$ & $3^{\circ} 01^{\prime} 22,25^{\prime \prime} 0$ & Vivarais \\
\hline $\mathrm{C} 3 \mathrm{C}$ & $\begin{array}{l}\text { Caserío Undio } \\
\text { Arriba } n^{\circ} 63\end{array}$ & $\begin{array}{l}\text { Bo Undio Arriba, } \\
\text { Zuhatza (Ayala) }\end{array}$ & $43^{\circ} 7^{\prime} 40,44^{\prime \prime} \mathrm{N}$ & $3^{\circ} 01 ' 22,35^{\prime \prime} 0$ & De José Blanco \\
\hline C3D & $\begin{array}{c}\text { Huerta caserío } \\
\text { Undio Arriba }{ }^{\circ} 63\end{array}$ & $\begin{array}{l}\text { Bo Undio Arriba, } \\
\text { Zuhatza (Ayala) }\end{array}$ & $43^{\circ} 7 ' 39,72 " \mathrm{~N}$ & $3^{\circ} 01 ' 21,78^{\prime \prime} 0$ & Folle Blanche \\
\hline C4A & Caserío Carrascal & Oquendo & $43^{\circ} 9^{\prime} 18,99^{\prime \prime} \mathrm{N}$ & $3^{\circ} 02^{\prime} 05,37^{\prime \prime} \mathrm{O}$ & Naparo \\
\hline C4B & Caserío Carrascal & Oquendo & $43^{\circ} 9^{\prime} 18,72^{\prime \prime} \mathrm{N}$ & $3^{\circ} 02^{\prime} 05,02^{\prime \prime} \mathrm{O}$ & CourbuBlanc \\
\hline C5A & Caserío Gomeztegi & Oquendo & $43^{\circ} 9^{\prime} 18,34^{\prime \prime} \mathrm{N}$ & $3^{\circ} 01^{\prime} 59,67$ "O & Mollar Cano \\
\hline C5B & Caserío Gomeztegi & Oquendo & $43^{\circ} 9^{\prime} 18,33^{\prime \prime} \mathrm{N}$ & $3^{\circ} 01^{\prime} 60,00 " 0$ & DucPetit \\
\hline $\mathrm{C} 5 \mathrm{C}$ & Caserío Gomeztegi & Oquendo & $43^{\circ} 9^{\prime} 18,18^{\prime \prime} \mathrm{N}$ & $3^{\circ} 01 ' 59,81$ "O & De José Blanco \\
\hline C6 & Caserío Unzabetxe I & Oquendo & $43^{\circ} 9^{\prime} 80,14^{\prime \prime} \mathrm{N}$ & $3^{\circ} 01^{\prime} 22,95^{\prime \prime} 0$ & $\begin{array}{l}\text { Moscatel de } \\
\text { Alejandría }\end{array}$ \\
\hline$C 7$ & Caserío Unzabetxe II & Oquendo & $43^{\circ} 9^{\prime} 60,04^{\prime \prime} \mathrm{N}$ & $3^{\circ} 01 ' 27,03^{\prime \prime} 0$ & Vivarais \\
\hline C8A & Caserío Sologuren & Quejana, Ayala & $43^{\circ} 4^{\prime} 49,91$ "N & $3^{\circ} 03^{\prime} 52,56 " 0$ & $\begin{array}{l}\text { Moscatel de } \\
\text { Alejandría }\end{array}$ \\
\hline C8B & Caserío Sologuren & Quejana, Ayala & $43^{\circ} 4^{\prime} 50,12^{\prime \prime} \mathrm{N}$ & $3^{\circ} 03^{\prime} 52,18^{\prime \prime} 0$ & Graciano \\
\hline C9A & $\begin{array}{l}\text { Cabaña frente } \\
\text { caserío el Tabor }\end{array}$ & Quejana, Ayala & $43^{\circ} 4^{\prime} 33,33^{\prime \prime} \mathrm{N}$ & $3^{\circ} 04^{\prime} 16,66^{\prime \prime} 0$ & Mollar Cano \\
\hline C9B & Caserío el Tabor & Quejana, Ayala & $43^{\circ} 4^{\prime} 33,39^{\prime \prime} \mathrm{N}$ & $3^{\circ} 04^{\prime} 17,01$ "O & Graciano \\
\hline C10 & Palacio de los Ayala & Quejana, Ayala & $43^{\circ} 4^{\prime} 34,18^{\prime \prime} \mathrm{N}$ & $3^{\circ} 04^{\prime} 24,48^{\prime \prime} 0$ & $\begin{array}{l}\text { Moscatel de } \\
\text { Estambul }\end{array}$ \\
\hline C11 & $\begin{array}{l}\text { Caserío Mesón } \\
\text { San Antón }\end{array}$ & $\begin{array}{l}\text { Bo Ibaizabal, } \\
\text { Llanteno, Ayala }\end{array}$ & $43^{\circ} 6^{\prime} 16,67 " N$ & $3^{\circ} 05^{\prime} 36,04^{\prime \prime} 0$ & Graciano \\
\hline C12 & $\begin{array}{c}\text { Cabaña del palacio } \\
\text { Isabel Novales }\end{array}$ & $\begin{array}{l}\text { Retes de Tudela, } \\
\text { Artziniega }\end{array}$ & $43^{\circ} 6^{\prime} 54,62^{\prime \prime} \mathrm{N}$ & $3^{\circ} 10^{\prime} 10,42^{\prime \prime} 0$ & Palomino Fino \\
\hline C13A & Casa beneficial & $\begin{array}{l}\text { Retes de Tudela, } \\
\text { Artziniega }\end{array}$ & $43^{\circ} 6^{\prime} 52,55^{\prime \prime} \mathrm{N}$ & $3^{\circ} 10^{\prime} 06,87^{\prime \prime} 0$ & $\begin{array}{l}\text { Moscatel de } \\
\text { Alejandría }\end{array}$ \\
\hline C13B & Casa beneficial & $\begin{array}{l}\text { Retes de Tudela, } \\
\text { Artziniega }\end{array}$ & $43^{\circ} 6^{\prime} 52,69^{\prime \prime} \mathrm{N}$ & $3^{\circ} 10^{\prime} 06,80^{\prime \prime} 0$ & $\begin{array}{l}\text { Moscatel de } \\
\text { Hamburgo }\end{array}$ \\
\hline
\end{tabular}




\begin{tabular}{|c|c|c|c|c|c|}
\hline $\begin{array}{l}\text { Código } \\
\text { muestra }\end{array}$ & $\begin{array}{l}\text { Denominación } \\
\text { muestra }\end{array}$ & $\begin{array}{l}\text { Pueblo/ } \\
\text { municipio }\end{array}$ & Latitud N & Longitud O & Identificación \\
\hline C14 & Plaza nº19 & $\begin{array}{l}\text { Retes de Tudela, } \\
\text { Artziniega }\end{array}$ & $43^{\circ} 6^{\prime} 52.58^{\prime \prime} \mathrm{N}$ & $3^{\circ} 10^{\prime} 05,23 " 0$ & Graciano \\
\hline C15 & $\begin{array}{l}\text { Casa de la parra cor- } \\
\text { tada (puerta verde) }\end{array}$ & $\begin{array}{l}\text { Retes de Tudela, } \\
\text { Artziniega }\end{array}$ & $43^{\circ} 6^{\prime} 51,19^{\prime \prime} \mathrm{N}$ & $3^{\circ} 10^{\prime} 05,69 " 0$ & Folle Blanche \\
\hline C16 & $\begin{array}{c}\text { Caserío } \\
\text { Inortzagarai } \\
\end{array}$ & Costera, Ayala & $43^{\circ} 6^{\prime} 11,23^{\prime \prime} \mathrm{N}$ & $3^{\circ} 06^{\prime} 17,18^{\prime \prime} 0$ & Graciano \\
\hline C17 & Caserío la Torre & $\begin{array}{c}\text { Santa Coloma, } \\
\text { Artziniega }\end{array}$ & $43^{\circ} 8^{\prime} 18,24 " \mathrm{~N}$ & $3^{\circ} 09^{\prime} 27,61$ "O & De José Blanco \\
\hline C18A & $\begin{array}{l}\text { La Viña (junto a } \\
\text { A-624) }\end{array}$ & $\begin{array}{l}\text { Santa Coloma, } \\
\text { Artziniega }\end{array}$ & $43^{\circ} 8^{\prime} 20,60^{\prime \prime} \mathrm{N}$ & $3^{\circ} 09^{\prime} 22,30^{\prime \prime} 0$ & Baco Noir \\
\hline C18B & $\begin{array}{c}\text { La Viña (junto a } \\
\text { A-624) }\end{array}$ & $\begin{array}{c}\text { Santa Coloma, } \\
\text { Artziniega }\end{array}$ & $43^{\circ} 8^{\prime} 20,60^{\prime \prime} \mathrm{N}$ & $3^{\circ} 09^{\prime} 22,30^{\prime \prime} \mathrm{O}$ & DucPetit \\
\hline C19A & $\begin{array}{c}\text { Huerta frente a la } \\
\text { casa (junto a A-624) }\end{array}$ & $\begin{array}{l}\text { Campijo Arriba, } \\
\text { Artziniega }\end{array}$ & $43^{\circ} 8^{\prime} 36,10^{\prime \prime} \mathrm{N}$ & $3^{\circ} 09^{\prime} 41,80^{\prime \prime} 0$ & De José Blanco \\
\hline C19B & $\begin{array}{c}\text { Huerta frente a la } \\
\text { casa (junto a A-624) }\end{array}$ & $\begin{array}{c}\text { Campijo Arriba, } \\
\text { Artziniega }\end{array}$ & $43^{\circ} 8^{\prime} 36,10^{\prime \prime} \mathrm{N}$ & $3^{\circ} 09^{\prime} 41,80^{\prime \prime} 0$ & De José Blanco \\
\hline C21A & Caserío landazuri & Inoso, Urkabustaiz & $43^{\circ} 0^{\prime} 33,24^{\prime \prime} \mathrm{N}$ & $2^{\circ} 56^{\prime} 09,15^{\prime \prime} 0$ & Tinto Velasco \\
\hline C21B & $\begin{array}{c}\text { Cabaña del } \\
\text { caserío Arana }\end{array}$ & Inoso, Urkabustaiz & $43^{\circ} 0^{\prime} 32,06^{\prime \prime} \mathrm{N}$ & $2^{\circ} 56^{\prime} 15,28^{\prime \prime} 0$ & Palomino Fino \\
\hline C21C & Caserío Arana & Inoso, Urkabustaiz & $43^{\circ} 0^{\prime} 31,74^{\prime \prime} \mathrm{N}$ & $2^{\circ} 56^{\prime} 14,55^{\prime \prime} 0$ & Graciano \\
\hline C21D & Caserío Arana & Inoso, Urkabustaiz & $43^{\circ} 0^{\prime} 31,75^{\prime \prime N}$ & $2^{\circ} 56^{\prime} 13,91$ "O & Graciano \\
\hline E1 & $\begin{array}{c}\text { Zona caserío } \\
\text { Araneko y circun- } \\
\text { valación de Amurrio }\end{array}$ & Amurrio & $43^{\circ} 2^{\prime} 32,20^{\prime \prime} \mathrm{N}$ & $2^{\circ} 59^{\prime} 24,30^{\prime \prime} \mathrm{O}$ & $\begin{array}{l}\text { Posible } \\
\text { silvestre }\end{array}$ \\
\hline E2 & $\begin{array}{c}\text { Entre C-625 y } \\
\text { río Nervión }\end{array}$ & Saratxo, Amurrio & $43^{\circ} 1 ' 32,30^{\prime \prime} \mathrm{N}$ & $3^{\circ} 00^{\prime} 33,30^{\prime \prime} 0$ & $\begin{array}{l}\text { Posible } \\
\text { silvestre }\end{array}$ \\
\hline E4 & $\begin{array}{l}\text { Puente de Iruleta } \\
\text { sobre río Izalde }\end{array}$ & Oquendo & $43^{\circ} 8^{\prime} 14,14^{\prime \prime} \mathrm{N}$ & $3^{\circ} 02^{\prime} 00.41^{\prime \prime} 0$ & $\begin{array}{l}\text { Posible } \\
\text { silvestre }\end{array}$ \\
\hline E5 & $\begin{array}{l}\text { Frente a casa con } \\
\text { cartel "Oquendo" }\end{array}$ & Oquendo & $43^{\circ} 8^{\prime} 48,29 " \mathrm{~N}$ & $3^{\circ} 02^{\prime} 02.21^{\prime \prime} 0$ & Baco Noir \\
\hline E6 & $\begin{array}{l}\text { La Viña (junto a } \\
\text { A-624) }\end{array}$ & $\begin{array}{l}\text { Santa Coloma, } \\
\text { Artziniega }\end{array}$ & $43^{\circ} 8^{\prime} 20,60^{\prime \prime} \mathrm{N}$ & $3^{\circ} 09^{\prime} 22,30^{\prime \prime} \mathrm{O}$ & $\begin{array}{l}\text { Posible } \\
\text { silvestre }\end{array}$ \\
\hline P1 & $\begin{array}{l}\text { Entre C-625 y } \\
\text { río Nervión }\end{array}$ & Saratxo, Amurrio & $43^{\circ} 1 ' 30,87^{\prime \prime N}$ & $3^{\circ} 00^{\prime} 33,75^{\prime \prime} 0$ & Desconocida \\
\hline
\end{tabular}

